\title{
Autoimmune liver diseases: internist's guide from bench to bedside
}

\author{
Mario Visconti \\ Department of Internal Medicine, Santa Maria del Popolo degli Incurabili Hospital, Naples, Italy
}

\begin{abstract}
Autoimmune liver diseases are disorders of unknown etiology and immune pathogenesis, characterized by liver parenchyma inflammation (autoimmune hepatitis) or by lesions of the intralobular biliary ducts (primary biliary cirrhosis) or of the entire biliary system (primary sclerosing cholangitis). They differ with regard to the epidemiological, clinical, morphological and serological features; the possible evolution; the different associations with other immune diseases of the digestive or extra-digestive organs; the treatment options. All progressively can result in hepatic cirrhosis. More recently, overlap syndromes have been identified, in which patients exhibit overlapping clinical, morphological and serological features of the above indicated diseases. The frequency of overlap syndromes is progressively increasing, causing additional clinical difficulties. Here, I review the diagnostic and clinical problems of the definite autoimmune liver diseases and of the overlap syndromes, with more regard to the evidences that drive current practice.
\end{abstract}

\section{Introduction}

Autoimmune liver diseases (AILDs) are disorders characterized by a not well-defined etiology and by an immune pathogenesis causing inflammatory lesions affecting the liver parenchyma, the small bile ducts or the entire biliary tract. They are classified in chronic autoimmune hepatitis (AIH), primary biliary cirrhosis (PBC) and primary sclerosing cholangitis (PSC). They differ with regard to the epidemiological, clinical, morphological and serological features; the possible evolution; the different associations with other immune diseases of the digestive or extra-digestive organs) (Table 1); ${ }^{1}$ the treatment options.

More recently, overlap syndromes (OS) have been identified with patients sharing clinical, morphological and serological features of the above in-

Correspondence: Visconti Mario, Rampe S. Giovanni Maggiore 12, 80134 Napoli, Italy.

Fax: +39.081.551539.

E-mail: mario.visconti1@tin.it

Key words: autoimmune liver diseases, autoimmune hepatitis, primary biliary cirrhosis, primary sclerosing cholangitis, overlap syndromes.

Received for publication: 8 April 2014.

Accepted for publication: 15 April 2014.

This work is licensed under a Creative Commons Attribution NonCommercial 3.0 License (CC BY-NC 3.0).

(C) Copyright M. Visconti, 2015

Licensee PAGEPress, Italy

Italian Journal of Medicine 2015; 9:20-36

doi:10.4081/itjm.2015.508 dicated diseases. The OS frequency is progressively increasing, causing additional diagnostic and therapeutic problems.

I, therefore, considered it worthwhile to briefly recap some basic knowledge of AILD and more extensively discuss the innovative aspects identified or defined in the more recent years, particularly focusing on the knowledge about the OS and on the current diagnostic and therapeutic strategies.

\section{Autoimmune chronic hepatitis}

The AIH can be defined as a hepatocellular inflammation sustained by hepatocyte immune tolerance loss, due to an unknown cause, and characterized by specific histological changes, hypergammaglobulinemia and serum autoantibodies positivity.

The key histological feature is interface hepatitis with portal and periportal lymphoplasmacytic infiltrates and hepatocellular necrosis. The severity of necroinflammatory activity is quite variable, ranging from mild hepatitis to massive hepatic necrosis. Cellular regeneration can result in the formation of rosette-like structures (Figure 1). Nodular regeneration and fibrosis with formation of portal-portal and portal-central bridges can result in cirrhosis.

\section{Epidemiology}

Autoimmune hepatitis is an infrequent, but not exceptionally rare, disease. The most reliable data about its frequency were collected in the Scandinavian countries, where the mean incidence is 1 to 2 per 100,000 inhabitants per year, and prevalence is 11 to 17 per 100,000 inhabitants. There is still a noticeable discrepancy between the frequency data col- 
lected in Norway in 1998 and those, well below, collected in Sweden in 2010. ${ }^{2,3}$ Similar incidence and prevalence can be assumed in most other countries, but in North America, where chronic viral hepatitis is less frequent, $\mathrm{AIH}$ represents $11-23 \%$ of chronic liver diseases.

Japanese $^{4}$ and, above all, Chinese ${ }^{5}$ clinical records would suggest that the disease is extremely rare in the Far East; however, more recent reports, based on more accurate epidemiological studies, indicate an increase of AIH in these countries. ${ }^{6}$

Women are more affected (70-80\% of cases), particularly between 15 and 40 years of age, ${ }^{7}$ but the disease can also occur in children and the elderly, including individuals older than $60 .{ }^{8}$ Of note, at the King's College Hospital of London tertiary pediatric liver center there has been a seven-fold increase in AIH incidence over the last decade. ${ }^{9}$

Table 1. Disease associations of autoimmune liver diseases.

\begin{tabular}{lll}
\hline AIH & PBC & PSC \\
\hline AI thyroiditis* & AI thyroiditis* & Ulcerative colitis* \\
Grave's disease & Rheumatoid arthritis* & Crohn's disease \\
Ulcerative colitis* & Sjögren's syndrome* & Colorectal cancer \\
Autoimmune emolytic anemia & Scleroderma/CREST syndrome & Celiac disease* \\
Idiopathic thrombocytopenia & Celiac disease* & Cholangiocarcinoma \\
Systemic lupus erythematosus & Mixed connective tissue disease & Rheumatoid arthritis* \\
Sjögren's syndrome* & Renal tubular acidosis & Histiocytosis X \\
Polymyositis & & Retroperitoneal fibrosis \\
Mixed connective tissue disease & & \\
Celiac disease* & &
\end{tabular}

AIH, autoimmune hepatitis; PBC, primary biliary cirrhosis; PSC, primary sclerosing cholangitis. *Possible association with several autoimmune liver diseases. Modified from Feld and Heathcote, 2003. ${ }^{1}$
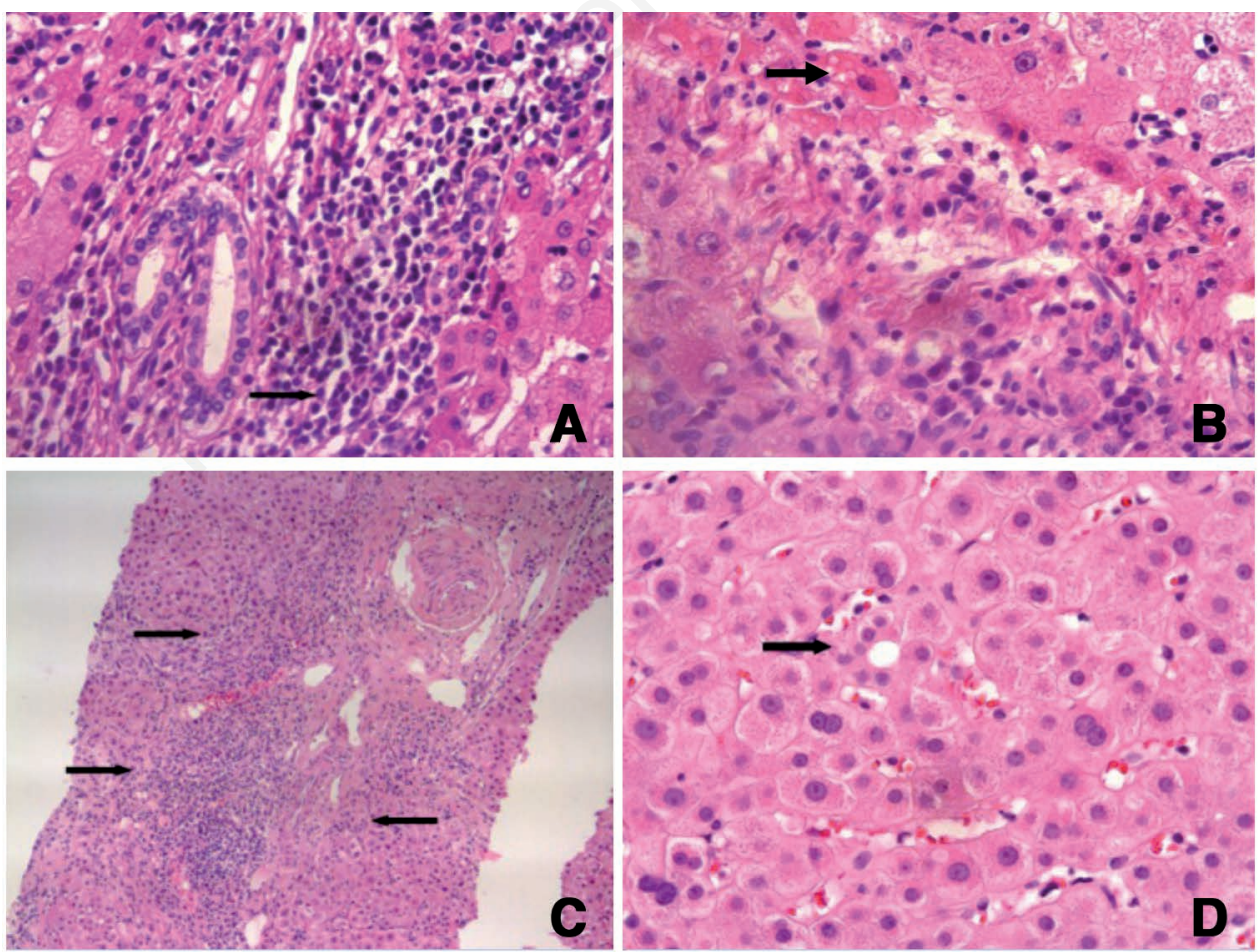

Figure 1. Histological features of autoimmune hepatitis. A) Inflammatory infiltrate with lymphocytes and a lot of plasmacells (arrow) (40X H\&E). B) Below: intralobular inflammatory infiltrate, predominantly plasmacells. Above: hepatocellular necrosis (arrow) (40X H\&E). C) Portal area considerably enlarged with severe inflammatory infiltrate, large interface hepatitis (20X H\&E). D) Rosette-like structure (40X H\&E). Courtesy of Giuseppina Marino Marsilia, A. Cardarelli Hospital, Naples. 


\section{Pathogenesis}

At least four factors likely contribute to the still not fully understood AIH pathogenesis: i) genetic predisposition related to the presence of some histocompatibility antigens (HLA A1, B8, DR3, DR4); ii) exposure on the cell membrane of autoantigens, as cytochrome P450 2D6 (CYP 2D6) and the asialoglycoprotein receptor (target antigens of anti-LKM1 and anti-ASGPR, respectively); iii) profound modification of the cellular immune response characterized by the secretion of various cytokines causing the differentiation of CD4 helper T-cells (Th0) in cytotoxic Th17, Th1 and Th2 cells. This results, with different mechanisms, in the activation of cells with direct cytotoxic effects and in the B-cell maturation into plasmacytes with the consequent production of autoantibodies; iv) role, still debated though, of potential triggers, both viral (HCV, HBV, cytomegalovirus, HSV) and nonviral (drugs as minocycline, statins, anti-TNF agent).

\section{Clinical features}

Children with AIH often present signs and symptoms of illness, similar to a viral hepatitis. In adults, both asymptomatic cases (up to $25 \%$ ), usually revealed only by a moderate increase in aminotransferases, and acute-onset cases (exceptionally fulminant) have been described.

In $40 \%$ of the cases the disease shows, since the beginning, chronic features characterized by nonspecific symptoms (fatigue, malaise, nausea, anorexia, weight loss, upper abdominal pain, arthromyalgia), differing, however, in severity. The association with other diseases, mainly autoimmune thyroiditis, diabetes, inflammatory bowel diseases, rheumatoid arthritis and amenorrhea, is frequent. Even the asymptomatic patients remain progressive and the diagnosis is often made when cirrhosis is already present.

\section{Laboratory abnormalities}

Elevated aminotransferases levels and hypergammaglobulinemia, mainly due to an increase in the immunoglobulin (Ig) G fraction, are characteristic AIH serological features. Cholestatic features are absent or less marked; thus, if they are prominent, a differential diagnosis with other diseases, such as viral hepatitis, drug-induced hepatopaties, PBC, PSC and OS, should be taken into consideration. ${ }^{10}$

As expected, when the disease progresses, typical laboratory abnormalities of cirrhosis (such as hypoalbuminemia and leuko-thrombocytopenia) may appear.

\section{Autoantibodies}

A characteristic mark of AIH is the serological presence of autoantibodies against nuclear components (ANA), smooth muscle (SMA), liver kidney microsome type 1 (anti-LKM1) and liver cytosol type 1 (anti-LC1). Based on autoantibodies, AIH can be classified in: type 1, positive for ANA and/or SMA, and type 2, positive for anti-LKM1 and anti-LC1. In North America 96\% of adult AIH patients are positive for ANA, SMA or both ${ }^{11}$ and $4 \%$ for anti-LKM1 and anti-LC $1 .{ }^{12}$ Anti-LKM1 are more frequent in European patients and are usually not accompanied by ANA or SMA (Figure 2). ${ }^{13,14}$

Clinical, biochemical and histological features are similar, but AIH 2 onset is more frequent during childhood, has a more severe presentation in young women and is more often associated with other autoimmune diseases. ${ }^{15}$

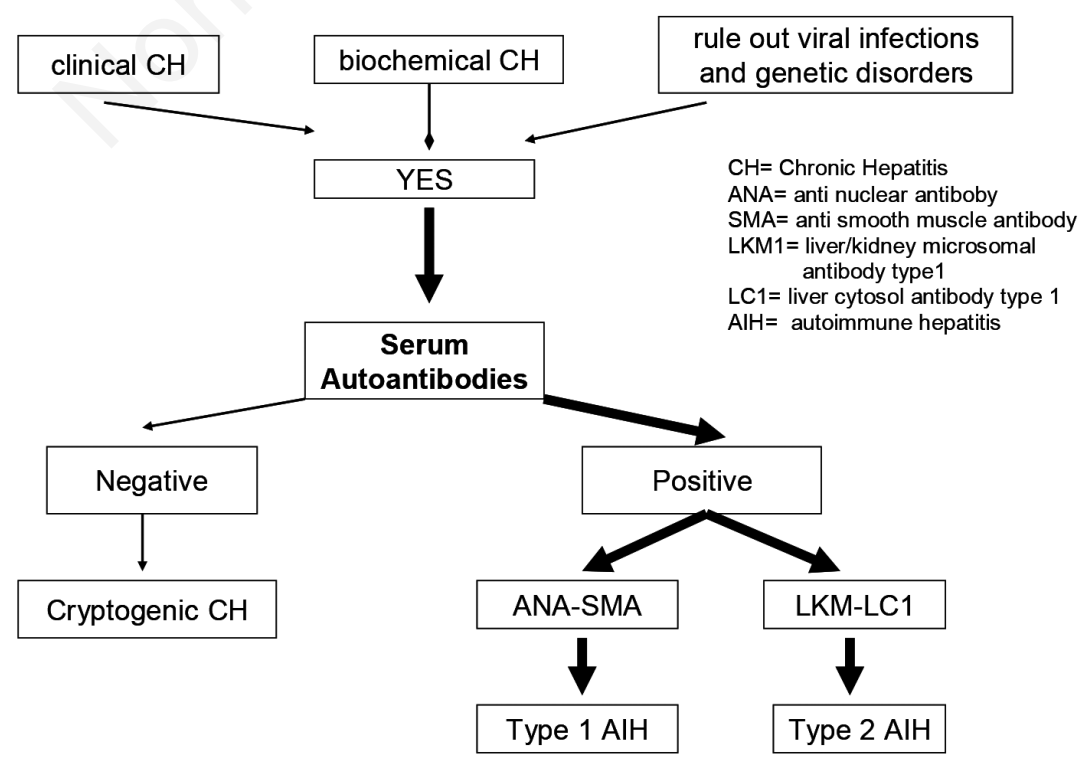

Figure 2. Flow chart for diagnosis and classification of autoimmune hepatitis. Modified from Bianchi et al., $2003 .{ }^{14}$ 
An AIH type 3, positive for anti-soluble liver antigen/liver-pancreas antigen (antiSLA/LPA), has also been described, but it is conceivable that these antibodies are a nonspecific sign of an AIH type 1, usually associated with a more severe clinical course. ${ }^{15}$ Also the antineutrophil cytoplasmic antibodies with perinuclear pattern (pANCA), described in AIH type 1, are non-specific AIH serological markers and their presence could be a sign of an AIH/PSC OS. Atypical pANCA react with peripheral nuclear membrane components, being for this reason named pANNA. ${ }^{9}{ }^{14}$ They can be detected in AIH type 1, but not in type 2, and further suggest an AIH diagnosis, particularly when other antibodies are absent. ${ }^{16}$ Despite their significance for AIH diagnosis and classification, the autoantibodies pathogenic role and the mechanisms by which they can possibly take part in liver damage still remain a topic for further research.

\section{Diagnosis}

AIH diagnosis may be challenging; however, early diagnosis is important because immunosuppressive therapy is life-saving. The International Autoimmune Hepatitis Group (IAIHG) codified in $1993^{17}$ and revised in $1999^{18}$ the diagnostic criteria and a diagnostic scoring system for $\mathrm{AIH}$, but diagnostic criteria were numerous (10) and complex and, therefore, meanly useful for scientific purposes. Thus, a further IAIHG study in 2008 aimed at the definition of more simplified diagnostic criteria for routine clinical practice. This retrospective cohort study included 359 AIH patients and 393 controls (training set and validation set). Patient data were collected from 11 international centers, all specialized in liver diseases, in 10 countries from North and South America, Europe, and Asia. Diagnostic criteria included sex, age, autoantibodies, immunoglobulins, absence of viral hepatitis, and histology (Table 2). ${ }^{19}$ The score has been shown to have a high degree of sensitivity and specificity for AIH diagnosis by three perspective studies. ${ }^{20-22}$

\section{Therapy}

Severe AIH is a potentially life-threatening disease without medication; thus, rapid diagnosis and institution of immunosuppressive therapy are mandatory. Despite successful treatment, cirrhosis can develop in many patients, being often already present at time of the diagnosis. The therapy is, however, still useful because it can reduce the activity of the disease..$^{14,23}$

Immunosuppressive treatment may be considered in asymptomatic adult patients with mild laboratory and histological changes, but the decision must be individualized and balanced against the possible side effects of the therapy.

Patients with minimal or no disease activity or inactive cirrhosis should not be treated, but must continue to be followed closely, i.e., 3-6 months. ${ }^{7}$

In severe AIH prednisone, starting with $30 \mathrm{mg}$ daily and tapering down to $10 \mathrm{mg}$ daily within 4 weeks, is the standard therapy for inducing remission. In many cases, the association with azathioprine, which can be instituted from the outset $(50 \mathrm{mg} /$ die in USA or $1-2 \mathrm{mg} / \mathrm{kg} /$ day in Europe) or within few weeks following steroid response, is preferred.

Monotherapy with a higher dose of prednisone, starting with 40-60 mg daily and tapering down to 20 $\mathrm{mg}$ daily within 4 weeks, can be preferred in patients with contraindications to azathioprine, in patients after 24 months of treatment, including those with cirrhosis. The 10 year-survival rate is $80 \%{ }^{24}$

Remission must be preserved by therapy with progressive reduction of steroid until $5 \mathrm{mg} /$ die and azathioprine $1-2 \mathrm{mg} / \mathrm{kg} / \mathrm{day}$, if in combination, or 2 $\mathrm{mg} / \mathrm{kg} /$ day, if alone. ${ }^{9}$

Table 2. Simplified diagnostic criteria for autoimmune hepatitis.

\begin{tabular}{lll}
\hline Variable & Cutoff & Points \\
\hline ANA or SMA & $\geq 1: 40$ & 1 \\
\hline ANA or SMA & $\geq 1: 80$ & $2 *$ \\
or LKM & $\geq 1: 40$ & \\
or SLA & Positive & 1 \\
\hline IgG & $>$ Upper normal limit & 2 \\
\hline Liver histology (evidence of hepatitis & $>1.10$ times upper normal limit & 1 \\
if a necessary condition) & Compatible with AIH & 2 \\
\hline Absence of viral hepatitis & Typical of AIH & 2 \\
\hline & & \\
& $\geq 6:$ probable AIH & \\
\hline
\end{tabular}

ANA, autoantibodies against nuclear components; SMA, smooth muscle; LKM, liver kidney microsome; IgG, immunoglobulin G; AIH, autoimmune hepatitis. *Addition of points achieved for all autoantibodies (maximum, 2 points). Modified from Hennes et al., 2008. ${ }^{19}$ 
Once remission (clinical, biochemical, immunological, histological) is achieved, prednisone dosage can be slowly reduced. Remission is achievable in around $80 \%$ of patients.

Maintenance treatment with steroids and azathioprine has not been proven to be more effective than azathioprine alone; ${ }^{25}$ however, histological remission of disease has been systematically evaluated only during follow up of patients in monotherapy with azathioprine. ${ }^{24,25}$ Nevertheless, tapering of steroids is commonly performed in clinical practice without repeating liver biopsy although relapse and progression to fibrosis are almost universal when immunosuppression is stopped in the presence of residual interface hepatitis. ${ }^{26-28}$ The combination of normal IgG and transaminase levels together correlates with lower histological inflammatory scores (Knodell index $<4$ ) 29 in around $90 \%$ of cases, although histological remission may persist 3-6 months behind biochemical remission. Treatment until normal liver alaninotransaminase (ALT), aspartate transaminase (AST), bilirubin and $\gamma$ globulin levels, and normal liver histology is ideal as this reduces the frequency of relapse after drug withdrawal from $86 \%$ to $60 \%$.

The ideal duration of therapy is debated, but for type1 AIH patients without cirrhosis a limited treatment with steroids for 12-18 months and azathioprine for 2-5 years is quite reasonable.

Relapses are very frequent, occurring in up to $80 \%$ of patients about 3 years after treatment withdrawal. They are associated with the possibility of cirrhosis and liver failure; if frequent, they increase the risk of hepatocarcinoma and all-cause mortality. ${ }^{30}$

Orthotopic liver transplantation is the obvious treatment of acute or chronic intractable liver failure. The outcome is very successful; a five-year survival is achieved in over $85 \%$ of cases. ${ }^{14}$

\section{Alternative treatments}

In patients non responders to standard therapy or with intolerance or low compliance to steroids alternative immunosuppressive drugs have been proposed, but all need further studies about theoretical more important positive or negative effects. ${ }^{9}$

Budenoside has a high affinity for the glucocorticoid receptors. It undergoes over $90 \%$ at hepatic first pass metabolism and its catabolities are devoid of corticoid activity. So steroid related adverse effects are limited.

In the early studies on small case series the results implied that, at least, budenoside was non inferior to prednisolone and many (58-83\%) complete remissions were achieved. ${ }^{31}$

Recently in a multicenter double-blind study 203 patients were randomized to trait with budenoside or prednisone, both in combination with azathioprine: after
6 months more biochemical remissions were obtained in budenoside arm $(60 \%$ vs $38.8 \%) .{ }^{32}$ An open-label extension was performed and patients of original prednisone arm were switched to receive budenoside; they achieved again more biochemical remission, if compared with the group originally randomized to budenoside $(68.2 \%$ vs $50.6 \%)$. These promising results must be confirmed, because the percentage of remissions with prednisone lower than the one obtained in historical case series and the histological data are necessary to prove a long-term complete and reliable remission.

In patients with cirrhosis and porto-systemic shunts the metabolic properties of the budenoside increase systemic availability and risks of adverse side effects. So budenoside cannot be employed for patients with cirrhosis and for all cases with severe liver function impairment. ${ }^{33}$ Moreover it has proven ineffective as a salvage drug for steroid-refractory or steroid dependent $\mathrm{AIH} .{ }^{34}$

Some encouraging results have been describe making use of other alternative drugs (mycophenolate mofetil, tacrolimus, cyclosporine, infliximab, cyclophosphamide, methotrexate), but they derive from small experiences or anedoctal reports. ${ }^{9}$ Mycophenolate mofetil is the most promising therapy, but at this moment many cases of intolerance to adverse side effects recommend the use only as empiric salvage therapy. ${ }^{35}$

\section{Primary biliary cirrhosis}

$\mathrm{PBC}$ is an idiopathic chronic cholestatic disease, resulting in the progressive destruction of the intrahepatic biliary ducts.

Liver biopsy allows distinguishing 4 histological stages during the course of PBC (Figures 3 and 4): ${ }^{36}$

- stage I, defined as floride bile-duct lesion, is characterized by inflammation and necrosis of the septal and interlobular ducts with focal and segmental distribution. Cholangiocytes may be vacuolated, shrunken, ballooning or picnotic. Around the biliary ducts is evident a lymphocytic infiltration; the damaged bile ducts can be centrally located in large granulomatous lesions consisting of lymphocytes, histiocytes, plasmacells, eosinophils and rare giant cells.

- In stage II (atypical duct proliferation) inflammation of the lesions is more diffuse, but less intense. Normal interlobular bile ducts disappear; atypical, poorly formed, tortuous and without lumen bile ducts are evident; inflammatory cells spill out of the portal triads and periportal hepatocytes are vacuolated and surrounded by macrophages (biliary piecemeal necrosis).

- In stage III (fibrosis) scars develop with formation of fibrotic bridges, adjoining the portal areas to 
some another and to centrolobular veins; however, nodular regeneration is not evident yet. The biliary ducts are greatly reduced and the cholestasis is already severe: many groups of hepatocytes contain a lot of lipidic or biliary material.

- In stage IV (cirrhosis) nodular regeneration is present. As typically observed in cholestasis, copper accumulates in the parenchyma and correlates with the serum bilirubin levels in the advancing states of disease. ${ }^{37}$

\section{Epidemiology}

The $\mathrm{PBC}$ incidence and prevalence are widely different among studies of specific defined populations. ${ }^{38}$ The highest annual incidence and point prevalence rates between 1980 and 2000 have been founded in: i) UK: incidence 3.1/100,000 inhabitants/year; preva- lence 25.1/100,000 inhabitants; ii) USA: incidence 2.7/100,000 inhabitants/year; prevalence 40.2/100,000 inhabitants.

In Norway and Sweden incidence and prevalence are higher than that reported in other populations, particularly in Asia, Canada and Australia.

In all countries PBC affects most frequently women, can affect all races, occurs between the fourth and sixth decades of life, is absent in children and accounts for between $0.5 \%$ and $2.0 \%$ deaths for cirrhosis.

\section{Pathogenesis}

The exact mechanism of liver damage is unknown, although many evidences indicate that it can be autoimmune. The data supporting this hypothesis are: abnormalities of the humoral and cellular immune systems (i.e., elevated serum immunoglobulin, mainly
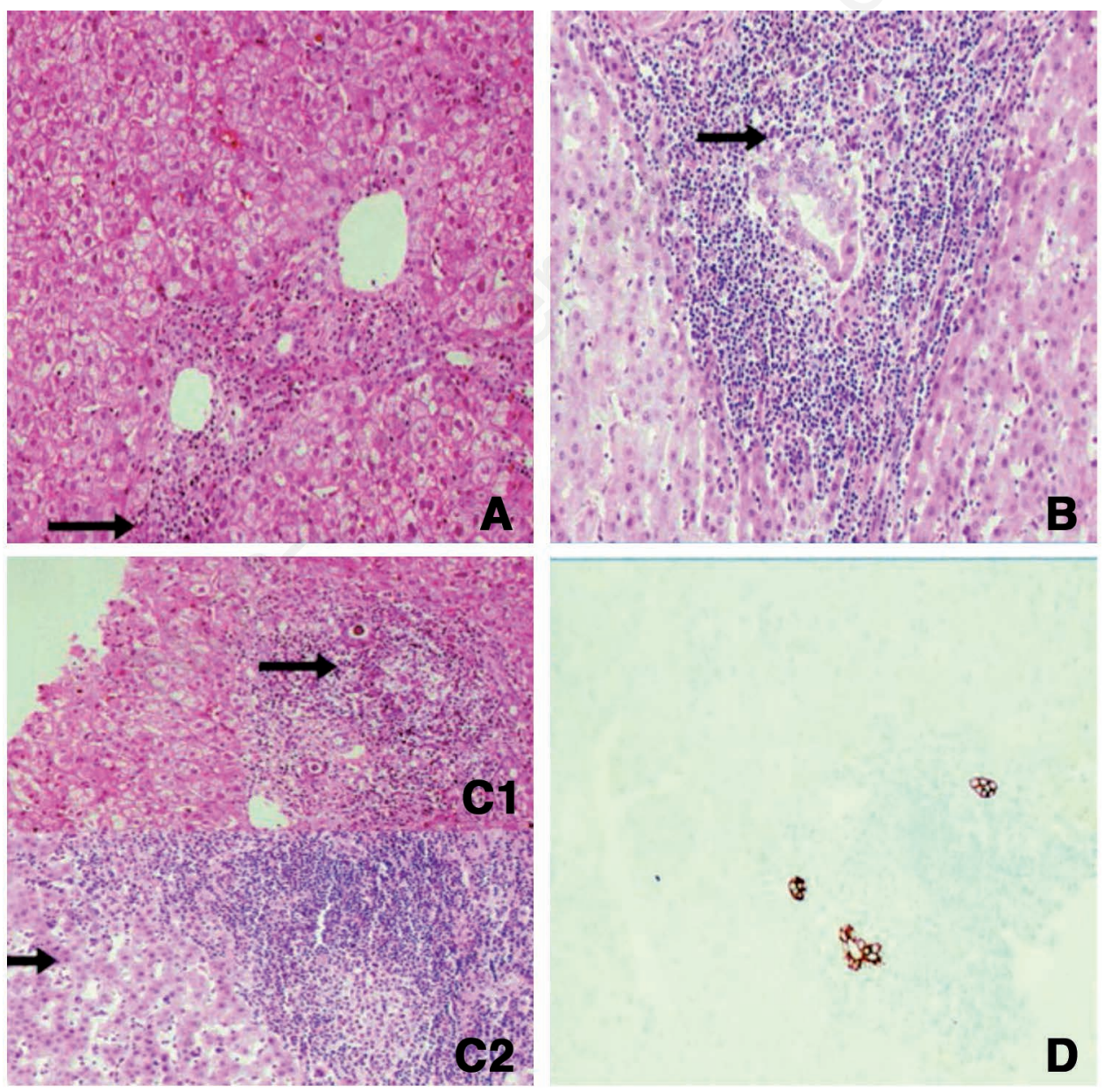

Figure 3. Histological features of primary biliary cirrhosis. A) Mild chronic inflammatory infiltrate surrounds bile duct (arrow). Stage I (20x H\&E). B) Lymphocytic aggregate surrounds a damaged duct with hyperplastic epithelium and disruption of basement membrane (arrow). Stage II (40x H\&E). C1) Epithelioid granuloma (arrow) (20X H\&E); C2) Epithelioid granuloma, the bile duct has disappeared. Left: foci regenerative, small hepatocytes (arrow) (40X H\&E). D) Keratin 7 shows a degenerate bile duct surrounded by an epithelioid granuloma. Courtesy of Giuseppina Marino Marsilia, A. Cardarelli Hospital, Naples. 
IgM, levels), multiple circulating autoantibodies, granulomas in the liver and regional lymph nodes, impaired regulation of both $\mathrm{B}$ and $\mathrm{T}$ lymphocytes and the association, as in AIH and PSC, with a variety of autoimmune-mediated diseases. ${ }^{39}$

Descriptive and epidemiological studies have confirmed three important distinct aspects contributing to the genesis of this chronic immunologically driven biliary disease. ${ }^{40}$ Firstly, PBC has an important genetic component, as demonstrated by the data showing an increased prevalence of PBC or other AILD in patients and their families. An association has been suggested between PBC and haplotype HLA-DR8 and, in some populations, HLA-DPB1.

The second feature is the probable role of environmental triggers such as smoking, recurrent urinary tract infections and possible chemical exposures. ${ }^{41}$

The third feature can be the abnormal expression of E2 mitochondrial antigens on the luminal surface of biliary epithelial cells.

Whatsoever the initial damage origin, activated CD4+ and CD8+ lymphocytes with a predominant Th1 response cause a continuous destruction of the interlobular biliary ducts. Once destroyed, the biliary duct regeneration is not possible or inefficient.

Subsequent to the intrahepatic bile duct loss, the normal bile flow is hampered with retention and deposition of the toxic substances normally excreted into the bile. The retention of toxic substances, such as bile acids and copper, can cause a further destruction of bile ducts and hepatocytes. In addition, increased HLA class II antigen expression occurs in the liver; so hepatocytes and bile duct epithelial cells are more susceptible to activated $\mathrm{T}$ lymphocytes and the immunologically mediated cytotoxicity is increased.

A controlled, interview-based study of 1032 pa- tients has confirmed many of the above described features. In genetically susceptible people environmental factors, including chemicals found in cigarette smoke and infectious agents introduced through urinary tract infections, may induce immune-mediated mechanisms, which cause PBC. The authors stated that exogenous estrogens may also contribute to the disease development, helping to explain why the disease occurs more frequently in females than in males. ${ }^{39,42}$

\section{Clinical features}

About $25 \%$ of $\mathrm{PBC}$ cases are diagnosed in asymptomatic patients during a routine blood evaluation.

In symptomatic patients the first reported symptom is often an inexplicable fatigue ( $65 \%$ of cases). Also pruritus $(55 \%)$ and right upper quadrant discomfort (8-17\%) can be early symptoms. Physical examination findings depend on the stage of the disease. In the early stages, examination findings are normal. As the disease advances, the following signs may be noted: hepatomegaly (25\%), hyperpigmentation (25\%), splenomegaly (15\%), jaundice $(10 \%)$, xanthelasmata $(10 \%)$.

In late stages of the disease is frequent a sicca syndrome $(50-75 \%)$ with xerophthalmia and xerostomia. Kayser-Fleischer rings are possible, but extremely rare.

In patients with advanced disease clinical signs of cirrhosis with its complications become evident.

\section{Diagnosis}

Diagnostic criteria for PBC are listed in Table $3 .{ }^{43}$

The cause of the importance of major criteria is the high specificity of the presence of antimitochondrial antibodies (AMA) in serum and of non-suppurative destructive cholangitis at histology. ${ }^{44,45}$

AMA in serum, particularly anti-M2 fraction (AMA

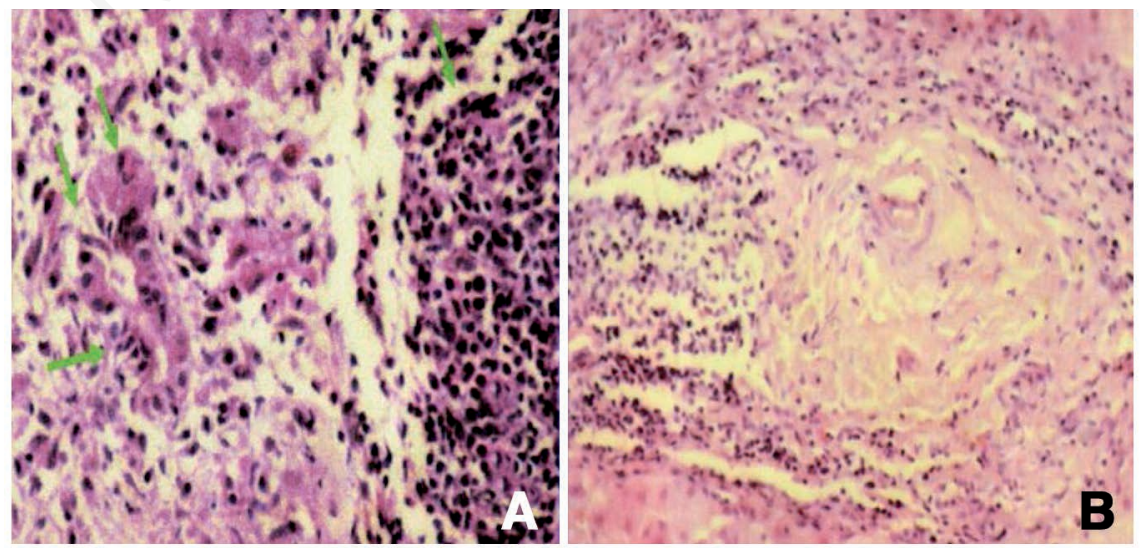

Figure 4. Histological features of primary biliary cirrhosis. A) Segmental damages of a biliary duct caused by the lymphocytic infiltration with disruption of basement membrane; some biliocytes are vacuolated; presence of a lymphomonocytic granuloma. Stage I (40X H\&E). B) Ductopenia: biliary duct replaced by fibrosis; adjacent granuloma. Stage II-III (40X H\&E). Courtesy of Giuseppe Pasquale, Second University of Naples. 
positivity by IF $>1: 40$ ), are the hallmark of $\mathrm{PBC}$. AMA can be found in $90-95 \%$ of $\mathrm{PBC}$ patients and have a specificity of $98 \%$ for this disease. Other fractions of AMA and autoantibodies (ANA, SMA, without a specific diagnostic significance) can be found in serum.

Some patients have clinical, biochemical, and histological features of $\mathrm{PBC}$, but their sera are negative for AMA. The diagnosis of autoimmune cholangitis has been used for these patients. However, even with the most sophisticated immunotesting, around $5 \%$ of patients remain AMA-negative. ${ }^{46}$

Other laboratory findings can become altered in PBC: i) increased aminotransferases and gammaglutamyltranspeptidase levels; ii) increased lipid and cholesterol levels, with an increased high-density lipoprotein fraction; iii) increased erythrocyte sedimentation rate.

Elevated bilirubin level, prolonged prothrombin time, and decreased albumin level indicate progression of the disease to cirrhosis. Thrombocytopenia indicates portal hypertension.

Imaging studies [abdominal ultrasonography, computed tomography scanning, or magnetic resonance imaging (MRN)] can be important to exclude biliary obstruction.

\section{Therapy}

The median survival of $\mathrm{PBC}$ untreated patients is approximately 9-10 years from presentation, with 26\% developing liver failure during this time. ${ }^{47}$ After a relatively stable phase, serum bilirubin increases sharply in the months preceding death. In patients with serum bilirubin levels above $2 \mathrm{mg} / \mathrm{dL}$, the mean survival is 4 years; in those with values above $6 \mathrm{mg} / \mathrm{dL}$, is 2 years. ${ }^{48}$

Ursodeoxycholic acid (UDCA) has demonstrated consistent evidence in improving liver biochemistry in PBC and patients receiving UDCA have a delayed rate of histological progression to cirrhosis. The destruction of interlobular biliary ducts causes retention of hydrofobic bile acids inside the hepatocytes, probably contributing to the progressive damage of hepatic function in PBC. UDCA reduces the level of hydrofobic bile acids inside the hepatocytes and may have also a protective effect on hepatic cellular membranes. UDCA may also limit the immune system alterations, by reducing the abnormal expression of HLA I and II antigens on the luminal surface of hepatocytes and biliary epithelial cells.

Many clinical studies have confirmed the benefits of UDCA therapy in $\mathrm{PBC}$, as patients receiving UDCA have a delayed rate of histological progression to cirrhosis. ${ }^{49,50}$

Patients with stage I/II PBC achieving an AST and alkaline phosphatase (ALP) less than $1.5 \times$ upper limit of normal (ULN) and normal bilirubin 12 months after starting UDCA therapy have an outcome free of liverrelated death, transplantation, progression to cirrhosis or liver failure. ${ }^{51}$

A combined analysis of three randomized-controlled trials, including 548 PBC patients, showed improved survival without liver transplantation in patients with moderate to severe disease treated with UDCA at doses of $13-15 \mathrm{mg} / \mathrm{kg} /$ day for up to 4 years. ${ }^{52}$

The degree of the biochemical response to UDCA identifies patients with a good long-term prognosis. ${ }^{53,54}$ Patients, showing ALP $<3$ ULN, AST $<2$ ULN, and bilirubin $61 \mathrm{mg} / \mathrm{dL}$ after 1 year of UDCA, had a 10 -year transplant-free survival rate of $90 \%$ (95\% confidence interval, $81-95 \%)$, compared to $51 \%(95 \%$ confidence interval, $38-64 \%$ ) for those who did not $(\mathrm{P}<0.001) .{ }^{55}$

Therefore UDCA, at the dose of $13-15 \mathrm{mg} / \mathrm{kg} /$ day, is currently considered the mainstay of therapy for $\mathrm{PBC}$ and all PBC patients with abnormal liver biochemistry should be considered for its utilization.

\section{Alternative and adjuvant therapies}

A large number or anti-inflammatory, immunosuppressive and anti-fibrotic drugs have been tested in PBC patients.

Glucocorticoids have been shown to provide benefits in patients treated with UDCA. A combination of

Table 3. Diagnostic criteria for primary biliary cirrhosis.

\author{
Major criteria \\ Presence of AMA with M2 specificity \\ Destruction of biliary ducts at histology

\section{Minor criteria} \\ Pruritus \\ Elevation of serum bilirubin level $>2 \mathrm{mg} / \mathrm{dL}$ \\ Elevation of serum alkaline phosphatase \\ Increase of IgM \\ Sjögren's syndrome
}

Definite $\mathrm{PBC}=2$ major criteria +2 minor criteria or 1 major criterion +4 minor criteria

Probable $\mathrm{PBC}=2$ major criteria or 1 major criterion +2 minor criteria

AMA, antimitochondrial antibodies; IgM, immunoglobulin M; PBC, primary biliary cirrhosis. Modified from Ideo, 2005. ${ }^{43}$ 
glucocorticoids (specifically budesonide) and UDCA leads to a better biochemical response and a better histological response in terms of inflammation and fibrosis in de novo PBC patients. ${ }^{56,57}$ Budenoside is not, however, suitable for cirrhotic patients.

No patients treated with methotrexate (MTX) in the precirrhotic stage at baseline showed progression to cirrhosis, suggesting that MTX could be useful in a small subset of patients. ${ }^{58}$

Colchicine improved tests of liver function in three prospective studies and was associated with improved survival for up to 4 years. However, survival benefits were lost after 8 years. Colchicine appears to slow the rate of $\mathrm{PBC}$ progression but not to stop it. ${ }^{59}$

Treatment with the combination of UDCA and MTX or UDCA and colchicine led to sustained clinical remission in a subset of PBC patients. The response to the UDCA and MTX combination appeared more durable than UDCA and colchicine. ${ }^{60}$

However, other studies have found no efficacy of methotrexate when used alone or in combination with UDCA. ${ }^{61,62}$

Randomized control trials of cyclosporine monotherapy in PBC have shown that the drug is effective in terms of clinical, biochemical and histological progression ${ }^{63}$ and significantly prolonged the time to death or transplantation, ${ }^{64}$ despite the well-known detrimental influence of cyclosporine on hepatobiliary transport systems.$^{65}$ However, because of the high rate of side effects, there has been little enthusiasm to further examine the potential benefit of cyclosporine, particularly in association with UDCA.

Approximately one-third of PBC patients may not respond to UDCA and, remaining at risk of progressive liver disease, are candidates for an alternative therapy.

Preliminary studies indicate that patients with a suboptimal response to UDCA and frank biliary and periportal inflammation benefit from the combination of UDCA and glucocorticoids (in particular budesonide) in terms of survival without liver transplantation. ${ }^{66}$

Methotrexate improved biochemical test results and liver histological findings when it was added to UDCA in patients who had an incomplete response to UDCA. ${ }^{67}$

These data make us consider many studies about the drugs already mentioned or in early experimental stage (i.e. $\alpha$ agonists, estrogen- $\alpha$ receptor agonists).

\section{Liver transplantation}

Liver transplantation is the only effective treatment for patients with decompensated cirrhosis or liver failure and for patients with severe destruction of bile ducts, greatly improving the patient survival. ${ }^{68}$ Transplantation may be indicated also in case of bilirubin $>6 \mathrm{mg} / \mathrm{dL}$, untreatable pruritus, severe osteoporosis, inappropriate quality of life. ${ }^{37}$

PBC relapses in about $20 \%$ of patients at 5 years. ${ }^{69}$

\section{Symptomatic therapy}

Pruritus: cholestyramine $(8-16 \mathrm{mg} / \mathrm{die})$ is the first therapy. Other therapies include rifampicin glucocorticoids, sertraline and opiate antagonists. Plasmapheresis, biliary, drainage or extracorporeal albumin dialysis ${ }^{70}$ may be successful when other treatments fail.

Hypercholesterolemia: UDCA induces an average $15-20 \%$ decrease in total and LDL cholesterol at 1 year of therapy. Also statins are safe and effective in PBC.

Osteopenia and osteoporosis: current treatments for osteopenia and osteoporosis include: physical activity, calcium and vitamin D supplementation, bisphosphonates or estrogens supplementation.

\section{Primary sclerosing cholangitis}

PSC is a chronic cholestatic disease of unknown etiology characterized by inflammation and subsequent fibrosis of the extra- and intrahepatic biliary ducts. PSC slowly progresses to biliary duct obstruction, secondary hepatic cirrhosis and hepatic failure. The lesions of the extra-hepatic ducts are prevalent or, in a small number of cases, exclusive. Persistent pathology of the only little biliary ducts is not compatible with $\mathrm{PBC}$ but recently have been described some cases with subsequent lesions of the extrahepatic ducts. ${ }^{71}$

\section{Epidemiology}

PSC prevalence and incidence rates differ worldwide. In North America and Europe prevalence ranges from 6 to 16 cases/100,000 inhabitants; incidence is about 1 case/100,000 inhabitants/year. ${ }^{72-74}$ Lower data are reported in Asia and Sothern Europe. ${ }^{75,76}$ PSC affects more frequently young males (about 30-40 years old) with a $2 / 1$ male/female ratio; among females the disease often affects a lightly higher age; sometimes also children can be affected. ${ }^{77}$

\section{Pathogenesis}

PSC is a complex disease. Pathogenesis is not well defined, many factors contributing to determine the anatomical and clinical features of the disease.

Genetic predisposition derived from the following items: i) increase in risk (9- to 39-fold) for the development of the disease in patients who have a first-degree relative with $\mathrm{PSC} ;{ }^{78}$ ii) frequent association with many HLA haplotypes, including HLA-DRB1*1501DQB1*0602, HLA-DRB1*1301-DQB1*0603, and HLA-A1-B8-DRB $1 * 0301-D Q B 1 * 0201 .{ }^{79-82}$ The HLA aplotype $\mathrm{B} 8, \mathrm{Dr} 3$, frequent also in $\mathrm{PBC}$, has been associated with a number of other autoimmune diseases such as AIH, thyroiditis, celiac disease and myasthenia gravis; iii) its association with inflammatory bowel disease (IBD), in particularly with ulcerative colitis (UC). 
To date, 12 non-major histocompatibility loci have been associated with PSC. Most of these loci have a stronger association with PSC than IBD, indicating the overlapping yet unique genetic architecture between PSC and IBD. ${ }^{83}$

Infections could be important in cases associated with UC, bacterial invasion of portal vein and lymphatic vessels, or enterohepatic translocation of endotoxins. ${ }^{84}$ However, studies in humans have suggested that portal venous bacteremia is uncommon in UC. ${ }^{85}$ The etiological involvement of viruses such as reovirus or cytomegalovirus has never been documented. In spite of these doubts about etiology, it is generally accepted an immune pathogenesis for PSC. Perinuclear antineutrophil cytoplasmic antibodies (pANCA) can be found in $26-85 \%$ of patients with PBC, where is often possible the serum presence of other autoantibodies, i.e. anticardiolipin, ANA and SMA. Cell-mediated immune abnormalities have been described including decreased circulating $\mathrm{T}$ cells, increase $\mathrm{CD} 4 / \mathrm{CD} 8$ ratio and increased number of $\mathrm{B}$ cells. The cellular immune response alterations could be caused by an overexpression of HLA class II antigens on the cellular membrane of the biliary ducts. ${ }^{37}$

\section{Clinical features}

The PSC natural history is more variable than that of AIH or PBC. Many patients (30-50\%) at diagnosis are young male with IBD who present serum cholestatic alterations. They could remain without symptoms for many years and have a median survival transplantation free of 12 years. ${ }^{86}$ Although asymptomatic, some patients may have advanced disease, as evaluated both histologically and radiologically. Symptomatic patients may present with fever, jaundice, pruritus, and right upper quadrant pain. In these patients PSC has a more progressive outcome and $90 \%$ with stage 2 (periportal disease) progressing over 5 years.

Because of the presence of bile duct strictures and the formation of biliary stones and sludge, PSC is commonly complicated by recurrent episodes of bacterial cholangitis, which can resolve spontaneously or need antibiotic treatment or endoscopic dilatation.

IBD is a common feature of PSC. Patients with PSC should undergo colonoscopy and biopsies to determine if they have IBD, even when there are no symptoms. The chronological order of diagnosis has switched in past decades. In a recent cohort (20032007), most patients were diagnosed with PSC first; in an earlier cohort (1993-1997), most patients were diagnosed with IBD first. ${ }^{87}$

$\mathrm{UC}$ is the most frequent clinical expression of IBD associated with PSC. Extension, histological inflammation and clinical features of UC in PSC patients may be very different and often of no similar severity. ${ }^{88}$

Patients with PSC and UC have an increased cu- mulative risk of colorectal cancer (CRC) compared with those with UC alone. PSC can be diagnosed after colectomy just as IBD can develop after liver transplantation. ${ }^{89,90}$ Similarly, patients diagnosed with PSC without IBD can still develop this disease and $\mathrm{CRC}$ at a later time. Thus, a colonoscopy every 5 years after the diagnosis of PSC to exclude or confirm the development of CRC or IBD has been suggested. ${ }^{91}$

The development of cholangiocarcinoma is a major complication, seen in up to $16 \%$ of PSC patients. PSC patients develop cholangiocarcinoma with a frequency of $6-11 \%$ in studies of natural history and of $7-36 \%$ in studies of patients who received liver transplantation. ${ }^{37}$ Cholangiocarcinoma is an early complication of PSC because $35 \%$ of the cases occur only 2 years after the diagnosis although some cases have been described even after 25 years. ${ }^{37}$

Accurate diagnosis of cholangiocarcinoma remains a problem in many cases, as tumor may be indistinguishable from stricture on cholangiogram and accurate cytologic diagnosis from bile duct brushings may be exceedingly difficult. Greatly elevated CA199 levels may be of utility, although can be observed a considerable overlap with PSC without cancer.

\section{Liver histology}

Liver biopsy is required in PSC patients only when is suspected an overlap AIH syndrome. PSC histological features are often non-specific and prone to sampling variations due to the heterogeneous involvement of the biliary tree. ${ }^{92}$ Unfortunately, the classic description of concentric ductal fibrosis (onion skinning) involving bile ducts within portal tract areas is rarely encountered in clinical practice. ${ }^{93}$ Non-invasive methods are useful to assess the entity of fibrosis and diagnosis of cirrhosis.

\section{Diagnosis}

PSC diagnosis is based on biochemical and imaging criteria (Figure 5). ${ }^{37} \mathrm{An}$ increase in alkaline phosphatase and gamma-glutammyl-transferases serum levels is the most common PSC biochemical abnormality. Although the levels can vary throughout the disease course seldom they are normal. ${ }^{94}$ Serum aminotransferase levels are frequently normal or slightly increased (about $\leq 2$ fold above the upper limit of normal), higher values suggesting an overlap syndrome with AIH. Total bilirubin serum levels are usually normal, strongly increasing only among patients with significant biliary obstruction.

Other abnormalities (i.e. hypoalbuminemia, prolonged prothrombin time, thrombocytopenia) are evident in patients with cirrhosis and its complications.

As stated above, autoantibodies (particularly pANCA) are present in serum of PSC patients. Some- 
times there are also increased levels of IgG-4, characterizing the association between IgG-4 associated cholangitis (IAC) and autoimmune pancreatitis (AIP).

Based on findings from retrospective studies, it is not clear whether some patients with PSC and increased serum levels of IgG4 actually have IAC/AIP or a subtype of PSC, characterized by a more aggressive disease course. ${ }^{95,96} \mathrm{~A}$ more precise diagnosis may be relevant because IAC/AIP patients should be considered for treatment with corticosteroids. ${ }^{97}$

MRN cholangiography (MRCP) is the technique of choice for identifying patients with PSC. The classic features include diffuse involvement of the extrahepatic and/or intrahepatic bile ducts, with multifocal annular structuring alternating normal or slightly dilated segments. However, up to $25 \%$ of patients have only intrahepatic disease. ${ }^{98} \mathrm{MRCP}$ is non-invasive, avoids radiation, is cost effective than endoscopic retrograde cholangio-pancreatography $(\mathrm{ERCP})^{99,100}$ and allow better diagnostic performance for detection of PSC, if compared with ERCP. With stronger magnetic fields and availability of 3-dimensional image MRCP reprocessing is useful to visualize third- and fourth order intrahepatic ducts which improve the imaging researches sensitivity when no extrahepatic biliary strictures are present.

\section{Therapy}

All randomized controlled trials of drugs to improve PSC or prevent its progression have produced negative results. UDCA is the most commonly studied agent. Perhaps it may improve biochemical and some histological parameters; but, as all other drugs, it is not effective for the disease outcome and in improving liver transplantation need or death time. A meta-analysis of 8 trials confirmed that treatment with UDCA did not slow the PSC progression. ${ }^{101}$

A European study, which used a dosage of 17-23 $\mathrm{mg} / \mathrm{kg} /$ die of UDCA did not show increased survival times of PSC patients compared with placebo. ${ }^{102}$

High UDCA dosages $(28-30 \mathrm{mg} / \mathrm{kg} / \mathrm{die})$, utilized in a North American study, increased the risk of disease progression 2-fold compared with placebo; study end points included cirrhosis, varices, cholangiocarcinoma, liver transplantation, or death. ${ }^{103}$

Furthermore, a high-dose of UDCA was associated with an increased CRC risk among UC patients. ${ }^{104}$ Thus in PSC high doses of UDCA have not to be utilized because of their toxicity; however, lower doses also need further evidences about the efficacy and the safety.

Immunosuppressant agents (i.e. corticosteroids, cyclosporine, azathioprine, methotrexate) have no role in the PSC treatment and are not recommended. ${ }^{105}$

The endoscopic expansion of dominant strictures (defined as a stenosis $\geq 1.5 \mathrm{~mm}$ in the common bile duct or $\geq 1 \mathrm{~mm}$ in the hepatic duct) by dilation alone or dilation and stent placement can provide long-term biliary drainage, reduces symptoms and can prevent complications. ${ }^{106}$ Bacterial infections are often found in bile of patients with dominant strictures; oral antibiotics for a minimum of 5 days after dilation and/or stenting can reduce the risk of cholangitis.

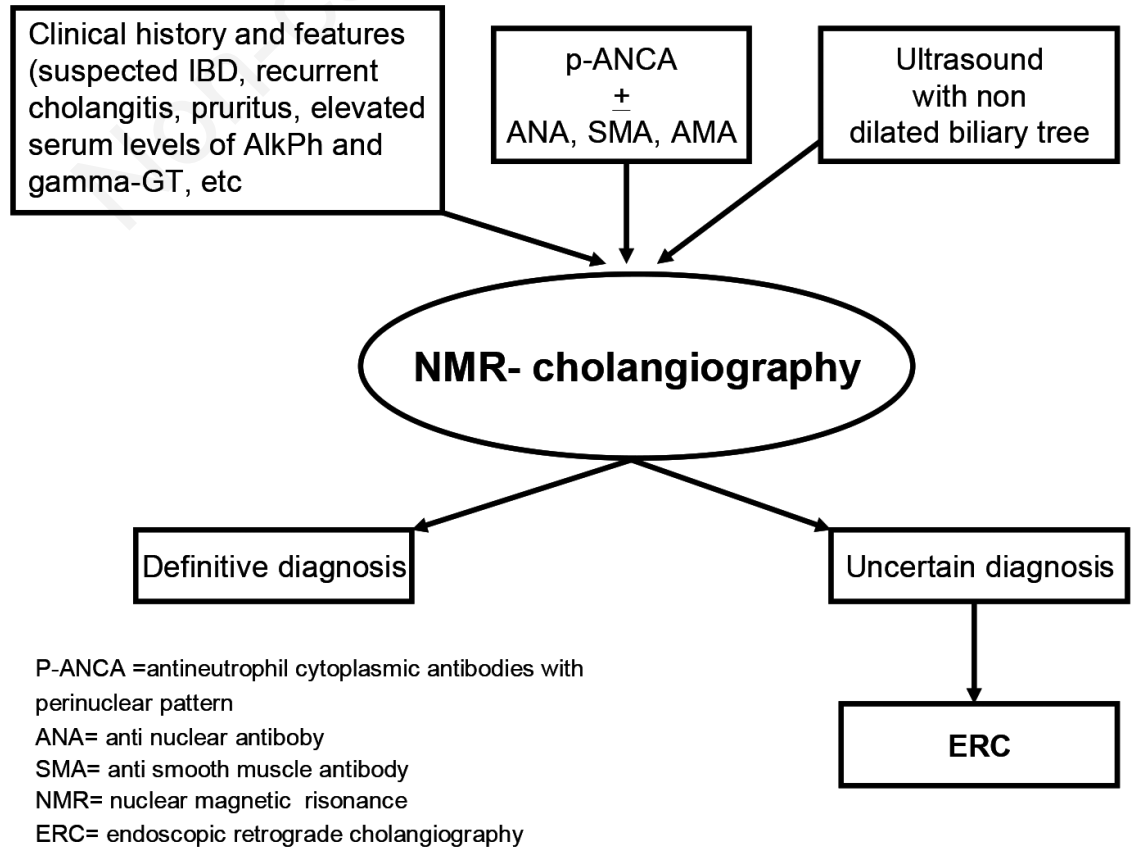

Figure 5. Flow chart for diagnosis of primary sclerosing cholangitis. Modified from Alvaro and Attili, $2003 .{ }^{37}$ 
Usually it is possible to remove stenting after 6-8 weeks, avoiding or reducing the risk of complications, above all of cholangitis. Nevertheless some patients require stenting with regular exchanges for about 612 months before the stricture resolves.

Liver transplantation gives very positive results in PSC. Patients with cirrhosis and pre-transplant ChildPugh-Turcotte class A have a survival rate higher than $90 \%$ at 5 years post-transplant. So, if possible, liver transplantation may be recommended in cirrhotics with a quite good model for end-stage liver disease scores and in non cirrhotics with untreatable recurrent episodes of cholangitis or inappropriate quality of life (i.e. impossibility to mitigate pruritus).

\section{Overlap syndromes}

The term overlap syndrome (OS) has been introduced to describe AILD variant forms which have both cholestatic and hepatitic features with overlapping characteristics of $\mathrm{AIH}+\mathrm{PBC}$ or $\mathrm{AIH}+\mathrm{PSC} .{ }^{107}$ Coexistence of $\mathrm{PBC}$ and $\mathrm{PSC}^{108}$ or $\mathrm{AIH}$ and both $\mathrm{PBC}$ and $\mathrm{PSC}^{109}$ have been reported in single cases, but there is no evidence for a possible overlap syndrome PBC/PSC. ${ }^{110}$

The OS incidence and prevalence have not been estimated yet. In a large study of the Mayo Clinic were evaluated 162 patient with $\mathrm{AIH}, 37$ with PBD and 26 with PSC; features of another AILD were found in $12 \%, 19 \%$ and $54 \%$, respectively. ${ }^{111}$ In our Department of Internal Medicine in Naples, where the AILD have lower incidence and prevalence than other hepatitis, during 5 years (1999-2003) we have observed OS biohumoral and histological features more often than the hepatic and biliary pure form features ( 9 cases: 5 OS, 4 pure forms). ${ }^{112}$

Also standardized definitions of OS are missed:

- It is unclear whether OS are either distinct autoimmune liver disorders or variants of AIH or attendant diseases affecting the same patient.

- The features of AIH and PBC or PSC usually occur simultaneously (termed as true OS), but they can also occur sequentially, i.e. the patients present with features of one disease and subsequently develop characteristics of the other. ${ }^{113}$ This is more frequent in AIH-PSC OS, where PSC can be diagnosed later, several years after the first features of AIH. ${ }^{114}$

- The clinical phenotypes of patients with the same overlap designation can be characterized by a considerable heterogeneity: ${ }^{115}$ i) an immunoserological overlap: positive ANA/ASMA titres and elevated $\operatorname{IgG}$ in conjunction with AMA-positive $\mathrm{PBC}$; ii) a biochemical overlap: AST/ALT $>5 \times \mathrm{ULN}$ in patients with $\mathrm{PBC}$ or PSC; or ALP $>3 \times \mathrm{ULN}$ in patients with AIH (or GGT $>5 \times \mathrm{ULN}$ in children); iii) a radiological overlap: clinical features of AIH with cholangiographic abnormalities indicative of an inflammatory cholangiopathy; iv) an histological overlap: lymphoplasmacytic infiltrate and interface hepatitis on liver biopsy with bile duct lesions indicative of either PBC or PSC. Diagnosis criteria for AIH-PBC have been suggested by Chazouillères et al. ${ }^{116}$ The diagnosis requires at least two out of three features for each component of the overlap. The PBC criteria comprise: i) ALP at least twice or GGT at least five times ULN; ii) positivity for AMA; and iii) histological evidence of florid bile duct lesions. The AIH criteria include: i) ALT at least 5 times ULN; ii) IgG at least 2 times ULN or positivity for SMA; and iii) liver biopsy with moderate or severe periportal or periseptal inflammation. ${ }^{116}$ Paris criteria have been confirmed by the European Association for the Study of the Liver (EASL) guidelines for the management of cholestatic liver diseases published in 2009, where it is, however, stressed that the histological evidence of interface hepatitis is essential for the diagnosis of PBC-AIH overlap. ${ }^{17}$

AIH-PSC overlap is characterized by ANA and/or SMA seropositivity, hypergammaglobulinemia, possible p-ANCA seropositivity, frequent concurrence of IBD, interface hepatitis and histological features typical of classical AIH in conjunction with cholestatic biochemical alterations and histological evolution to fibrous obliterative cholangitis, ductopenia, portal tract edema and/or bile stasis. The diagnosis is supported by cholangiographic changes (MRCP) of intrahepatic and/or extra-hepatic PSC. ${ }^{118,119}$

The association between PSC and AIH appears to be the most common OS. In a retrospective study, a high proportion of adults initially diagnosed as having AIH-1 were found to have sclerosing cholangitis on magnetic resonance cholangiography. ${ }^{120}$

The AIH-PSC OS is particularly frequent among children, as reported in Canadian and English series, ${ }^{121}$ therefore the term autoimmune sclerosing cholangitis (ASC) has been coined. In a prospective study conducted over 16 years all children with serological positive autoantibodies and high $\operatorname{IgG}$ levels and histological interface hepatitis features of AIDL underwent a cholangiogram at the time of presentation. Approximately half of them had bile ducts changes and were diagnosed as having ASC. All ASC patients were seropositive for ANA and/or SMA. ASC affects equally boys and girls. Children with ASC more commonly had concurrent inflammatory bowel disease and more often were positive for pANNA compared to those with AIH. ${ }^{122}$

The AIH OS treatment is reviewed in some recent articles. ${ }^{110,123}$

In AIH-PBC OS the treatment has to be guided by the clinical and /or histological aspects of the disease.

UDCA in monotherapy (13-15 mg/kg/die) may be effective in some patients with predominant features 
of PBC; these patients may present an adequate biochemical response and a slow progression of fibrosis and liver failure. ${ }^{124,125}$

Instead patients with evident signs of AIH (i.e. elevated serum transaminases and/or IgG levels, interface hepatitis) need immunosuppressive therapy (corticosteroid alone or plus azathioprine) in addition to UDCA in order to obtain a complete biochemical response. ${ }^{126}$ Alternatively it is possible to await results of the therapy with UDCA alone and then to consider the addition of immunosuppressives. Combination therapy is mandatory in patients with AIH and very high level of serum ALP, to obtain clinical remission and to reduce the development of fibrosis and the risk of hepatocellular carcinoma or cholangiocarcinoma. ${ }^{15,, 126,127}$

Positive responses of the biochemical features and/or fibrosis progression to the combination therapy in $\mathrm{AIH} / \mathrm{PBC}$ overlap have also been reported by others in retrospective or perspective studies, ${ }^{125,127,128}$ although the overall frequency of non-responders and the possibility of an unfavorable outcome of the disease are greater than those observed in AIH alone. ${ }^{128}$ Thus it still remains unclear whether the clinical outcome of AIH/PBC overlap is different to that of isolated $\mathrm{AIH}$ or $\mathrm{PBC}$.

The AIH-PSC OS prognosis may be better than that of PSC patients, but worse than that of patients with AIH alone. ${ }^{114}$

Remission of AIH-PSC OS with corticosteroids and azathioprine, sometimes combined with UDCA, is obtained in a small number of cases. The response to the immunosuppressive treatment appears to be better in children than in adults, probably because hepatitic features are more accentuated than biliary alterations. ${ }^{129,130}$

The treatment with high doses of UDCA (20-30 $\mathrm{mg} / \mathrm{kg} / \mathrm{die}$ ) have given strongly controversial results. In some studies these doses were thought to increase clinical benefit, decrease histological progression, and produce a preventive effect against colon cancer in IBD ${ }^{131,132}$ nevertheless, other recent data showed that UDCA not only is insufficient to favorably influence the disease progression and the cholangiocarcinoma development but it could also cause a direct hepatotoxic effect; ${ }^{101}$ also the preventive effect against colon cancer in IBD is doubtful. ${ }^{133}$

Usually AIH/PSC patients have a reduced survival compared with patients with $\mathrm{AIH}$ alone; ${ }^{128}$ however, patients with isolated PSC have a longer survival. ${ }^{134}$

It is unsurprising that AIH/PSC OS patients have a poorer outcome than patients with $\mathrm{AIH}$ alone and that the immunosuppressive therapy is often ineffective, ${ }^{135}$ because a very efficient pharmacologic therapy is lacking in PSC.

Liver transplantation must be considered in the late stages of both AIH-PBC and AIH-PSC OS.

\section{References}

1. Feld JJ, Heathcote EJ. Epidemiology of autoimmune liver disease. J Gastroenterol Hepatol 2003;18:1118-28.

2. Boberg KM, Aadland E, Jahnsen J, et al. Incidence and prevalence of primary biliary cirrhosis, primary sclerosing cholangitis, and autoimmune hepatitis in a Norwegian population. Scand J Gastroenterol 1998;33: 99-103.

3. Werner M, Prytz H, Ohlsson B, et al. Epidemiology and the initial presentation of autoimmune hepatitis in Sweden: a nationwide study. Scand J Gastroenterol 2008; 43:1232-40.

4. Toda G, Zeniya M, Watanabe F, et al. Present status of autoimmune hepatitis in Japan correlating the characteristics with international criteria in an area with a high rate of HCV infection. Japanese National Study Group of Autoimmune Hepatitis. J Hepatol 1997;26:1207-12.

5. Lam KC, Lai CL, Wu PC, Todd D. Etiological spectrum of liver cirrhosis in the Chinese. J Chronic Dis 1980;33:375-81.

6. Qiu D, Wang Q, Wang H, Xie Q, et al. Validation of the simplified criteria for diagnosis of autoimmune hepatitis in Chinese patients. J Hepatol 2011;54:340-7.

7. Manns MP, Czaja AJ, Gorham JD, et al. Diagnosis and management of autoimmune hepatitis. AASLD practice guidelines. Hepatology 2010;51:2193-213.

8. Al-Chalabi T, Boccato S, Portmann BC, et al. Autoimmune hepatitis (AIH) in the elderly: a systematic retrospective analysis of a large group of consecutive patients with definite AIH followed at a tertiary referral centre. J Hepatol 2006;45:575-83.

9. Liberal L, Grant CR, Mieli-Vergani G, Vergani D. Autoimmune hepatitis. A comprehensive review. J Autoimmun 2013;41:126-39.

10. Krawitt EL. Autoimmune hepatitis. N Engl J Med 2006;354:54-66.

11. Czaja AJ. Behavior and significance of autoantibodies in type 1 autoimmune hepatitis. J Hepatol 1999;30:394-401.

12. Martini E, Abuaf N, Cavalli F, et al. Antibody to liver cytosol (anti-LC1) in patients with autoimmune chronic active hepatitis type 2. Hepatology 1988;8:1662-6.

13. Homberg JC, Abuaf N, Bernard O, et al. Chronic active hepatitis associated with anti liver/kidney microsome type 1: a second type of "autoimmune" hepatitis. Hepatology 1987;7:1333-9.

14. Bianchi FB, Muratori P, Muratori L. Autoimmunity and autoreactivity in liver disease. In: Ascione A, ed. Hepatology at bedside. Napoli: Arte tipografica; 2003. pp 141-149.

15. Liberal R, Grant Cr, Longhi MS, et al. Diagnostic criteria of autoimmune hepatitis. Autoimmun Rev 2014 [Epub ahead of print].

16. Vergani D, Alvarez F, Bianchi FB, et al. Liver autoimmune serology: a consensus statement from the committee for autoimmune serology of the International Autoimmune Hepatitis Group. J Hepatol 2004;41:677-83.

17. Johnson PJ, McFarlaine IG. Meeting report: International autoimmune hepatitis group. Hepatology 1993; 18:998-1005.

18. Alvarez F, Berg PA, Bianchi FB, et al. International Autoimmune Hepatitis Group Report: review of criteria 
for diagnosis of autoimmune hepatitis. J Hepatol 1999; 31:929-38.

19. Hennes EM, Zeniya M, Czaja AJ, et al. Simplified criteria for the diagnosis of autoimmune hepatitis. Hepatology 2008;48:169-76.

20. Czaja AJ. Performance parameters of the diagnostic scoring systems for autoimmune hepatitis. Hepatology 2008;48:1540-8.

21. Muratori P, Granito A, Pappas G, Muratori L. Validation of simplified diagnostic criteria for autoimmune hepatitis in Italian patients. Hepatology 2009;49:1782-3.

22. Yeoman AD, Westbrook RH, Al-Chalabi T, et al. Diagnostic value and utility of the simplified International Autoimmune Hepatitis Group (IAIHG) criteria in acute and chronic liver disease. Hepatology 2009; 50:538-45.

23. Trived BJ, Hirschfield G. Treatment of autoimmune liver disease. Current and future therapeutic options. Ther Adv Chronic Dis 2013;4:119-41.

24. Montano-Loza A, Carpenter H, Czaja AJ. Consequences of treatment withdrawal in type 1 autoimmune hepatitis. Liver Int 2007;27:507-15.

25. Johnson P, McFarlane I, Williams R. Azathioprine for long-term maintenance of remission in autoimmune hepatitis. N Engl J Med 1995;333:958-63.

26. Stellon AJ, Keating JJ, Johnson P, et al. Maintenance of remission in autoimmune chronic active hepatitis with azathioprine after corticosteroid withdrawal. Hepatology 1988;8:781-4.

27. Carpenter H, Czaja AJ. The role of histological evaluation in the diagnosis and management of autoimmune hepatitis and its variants. Clin Liver Dis 2002;6:685-705.

28. Czaja AJ, Carpenter H. Histological features associated with relapse after corticosteroid withdrawal in type 1 autoimmune hepatitis. Liver Int 2003;23:116-23.

29. Lüth S, Herkel J, Kanzler S, et al. Serologic markers compared with liver biopsy for monitoring disease activity in autoimmune hepatitis. J Clin Gastroenterol 2008;42:926-30.

30. Yoshizawa K, Matsumoto A, Ichijo T, et al. Long-term outcome of Japanese patients with type 1 autoimmune hepatitis. Hepatology 2012;56:668-76.

31. Danielsson A, Prytz H. Oral budesonide for treatment of autoimmune chronic active hepatitis. Aliment Pharmacol Ther 1994;8:585-90.

32. Manns M, Woynarowski M, Kreisel W, et al. Budesonide induces remission more effectively than prednisone in a controlled trial of patients with autoimmune hepatitis. Gastroenterology 2010;139:1198-206.

33. Mederacke I, Helfritz F, Puls, F, et al. Budd-Chiari syndrome after treatment with budesonide in a cirrhotic patient with autoimmune hepatitis. Ann Hepatol 2012;11: 143-4.

34. Czaja AJ, Lindor K. Failure of budesonide in a pilot study of treatment-dependent autoimmune hepatitis. Gastroenterology 2000;119:1312-6.

35. Hlivko J, Shiffman M, Stravitz R, et al. A single center review of the use of mycophenolate mofetil in the treatment of autoimmune hepatitis. Clin Gastroenterol Hepatol 2008;6:1036-40.

36. Schaffner F, Popper H. Clinical-pathologic relations in primary biliary cirrhosis. Prog Liver Dis 1982;7:529-54.

37. Alvaro D, Attili AF. Biliary diseases: the cholan- giopathies. In: Ascione A, ed. Hepatology at bedside. Napoli: Arte tipografica; 2003. pp 327-346.

38. Field JJ, Heathcote EJ. Epidemiology of autoimmune liver disease. J Gastroenterol Hepatol 2003;18:1118-28.

39. Solis Herruzo JA, Solis Munoz P, Munoz Yague T. The pathogenesis of primary biliary cirrhosis. Rev Esp Enferm Dig 2009;101:413-23.

40. Hirschfield GM, Gershwin MR. Primary biliary cirrhosis: one disease with many faces. IMAJ 2011;13:55-9

41. Gershwin ME, Selmi C, Worman HJ, et al. Risk factors and comorbidities in primary biliary cirrhosis: a controlled interview-based study of 1032 patients. Hepatology 2005;42:1194-202.

42. McNally RJ, Ducker S, James OF. Are transient environmental agents involved in the cause of primary biliary cirrhosis? Evidence from space-time clustering analysis. Hepatology 2009;50:1169-74.

43. Ideo G. Le epatopatie autoimmuni e colestatiche. In: Ideo G, ed. Compendio di epatologia. Milano: Fondazione Amici Epatologia; 2005. pp 98-110.

44. European Association for the Study of the Liver. EASL Clinical Practice Guidelines: management of cholestatic liver diseases. J Hepatol 2009;51:237-67.

45. Lindor KD, Gershwin ME, Poupon R, et al. Primary biliary cirrhosis. Hepatology 2009;50:291-308.

46. Oertelt S, Rieger R, Selmi C, et al. A sensitive bead assay for antimitochondrial antibodies: chipping away at AMA-negative primary biliary cirrhosis. Hepatology 2007;45:659-65.

47. Prince M, Chetwynd A, Newman W, et al. Survival and symptom progression in a geographically based cohort of patients with primary biliary cirrhosis: follow-up for up to 28 years. Gastroenterology 2002;123:1044-51.

48. Shapiro JM, Smith H, Shaffner F. Serum bilirubin: a prognostic factor in primary biliary cirrhosis. Gut 1979;20:137-40.

49. Poupon R. Primary biliary cirrhosis: a 2010 update. J Hepatol 2010;52:745-58.

50. Trivedi PJ, Hirschfield G. Treatment of autoimmune liver disease. Current and future therapeutic options. Ther Adv Chronic Dis 2013;4:119-41.

51. Corpechot C, Chazouillères O, Poupon R. Early primary biliary cirrhosis: biochemical response to treatment and prediction of long-term outcome. J Hepatol 2011;55: 1361-7.

52. Poupon RE, Lindor KD, Cauch-Dudek K, et al. Combined analysis of randomized controlled trials of ursodeoxycolic acid in primary biliary cirrhosis. Gastroenterology 1997;113:884-90.

53. Pares A, Caballeria L, Rodes J. Excellent long-term survival in patients with primary biliary cirrhosis and biochemical response to ursodeoxycholic acid. Gastroenterology 2006;130:715-20.

54. Kuiper EM, Hansen BE, de Vries RA, et al. Improved prognosis of patients with primary biliary cirrhosis that have a biochemical response to ursodeoxycholic acid. Gastroenterology 2009;136:1281-7.

55. Corpechot C, Abenavoli L, Rabahi N, et al. Biochemical response to ursodeoxycholic acid and long-term prognosis in primary biliary cirrhosis. Hepatology 2008;48:871-7.

56. Leuschner M, Maier KP, Schlichting J, et al. Oral budesonide and ursodeoxycholic acid for treatment of 
primary biliary cirrhosis: results of a prospective double-blind trial. Gastroenterology 1999;117:918-25.

57. Rautiainen H, Karkkainen $\mathrm{P}$, Karvonen $\mathrm{AL}$, et al. Budesonide combined with UDCA to improve liverhistology in primary biliary cirrhosis: a three-year randomized trial. Hepatology 2005;41:747-52.

58. Kaplan MM, Cheng S, Price LL, Bonis PA. A randomized controlled trial of colchicine plus ursodiol versus methotrexate plus ursodiol in primary biliary cirrhosis: ten-year results. Hepatology 2004;39:915-23.

59. Kaplan MM. The use of methotrexate, colchicine, and other immunomodulatory drugs in the treatment of primary biliary cirrhosis. Sem Liver Dis 1997;17:129-36.

60. Leung J, Bonis PA, Kaplan MM. Colchicine or methotrexate, with ursodiol, are effective after 20 years in a subset of patients with primary biliary cirrhosis. Clin Gastroenterol Hepatol 2011;9:776-80.

61. Gonzalez-Koch A, BrahmJ, Antezana C, et al. The combination of ursodeoxycholic acid and methotrexate for primary biliary cirrhosis is not better than ursodeoxycholic acid alone. J Hepatol 1997;27:143-9.

62. Combes B, Emerson SS, Flye NL, et al. Methotrexate (MTX) plus ursodeoxycholic acid (UDCA) in the treatment of primary biliary cirrhosis. Hepatology 2005;42:1184-93.

63. Wiesner RH, Ludwig J, Lindor KD, et al. A controlled trial of cyclosporine in the treatment of primary biliary cirrhosis. N Engl J Med 1990;322:1419-24

64. Lombard M, Portmann B, Neuberger J, et al. Cyclosporin treatment in primary biliary cirrhosis: results of a longterm placebo controlled trial. Gastroenterology 1993;104:519-26.

65. Mita S, Suzuki H, Akita H, et al. Inhibition of bile acid transport across $\mathrm{Na}+$ /taurocholate cotransporting polypeptide (SLC10A1) and bile salt export pump (ABCB 11)-coexpressing LLC-PK1 cells by cholestasisinducing drugs. Drug Metab Dispos 2006;34:1575-181.

66. Poupon R. Primary biliary cirrhosis: a 2010 update. J Hepatol 2010;52;745-58.

67. Babatin MA, Sanai FM, Swain MG. Methotrexate therapy for the symptomatic treatment of primary biliary cirrhosis patients, who are biochemical incomplete responders to ursodeoxycholic acid therapy. Aliment Pharmacol Ther 2006;24:813-20.

68. Tzakis AG, Carcassonne C, Todo S, at al. Liver transplantation for primary biliary cirrhosis. Semin Immunol 1989;9:144-8.

69. MacQuillan GC, Neuberger J. Liver transplantation for primary biliary cirrhosis. Clin Liver Dis 2003;7:941-56.

70. Peres A, Cisneros L, Salmeron JM, et al. Extracorporeal albumin dialysis: a procedure for prolonged relief of intractable pruritus in patients with primary biliary cirrhosis. Am J Gastroenterol 2004;99:1105-10.

71. Eaton JE, Talwalkar JA, Lazaridis KN, et al. Pathogenesis of primary sclerosing cholangitis and advances in diagnosis and management. Gastroenterology 2013; 145:521-36

72. Lindkvist B, Benito de Valle M, Gullberg B, et al. Incidence and prevalence of primary sclerosing cholangitis in a defined adult population in Sweden. Hepatology 2010;52:571-7.

73. Bambha K, Kim WR, Talwalkar JA, et al. Incidence, clinical spectrum, and outcomes of primary sclerosing cholangitis in a United States community. Gastroenterology 2003;125:1364-9.

74. Molodecky NA, Kareemi H, Parab R, et al. Incidence of primary sclerosing cholangitis: a systematic review and meta-analysis. Hepatology 2011;53:1590-9.

75. Ang TL, Fock KM, Ng TM, et al. Clinical profile of primary sclerosing cholangitis in Singapore. J Gastroenterol Hepatol 2002;17:908-13.

76. Escorsell A, Pares A, Rodes J, et al. Epidemiology of primary sclerosing cholangitis in Spain. Spanish Association for the Study of the Liver. J Hepatol 1994;21:787-91.

77. Kaplan GG, Laupland KB, Butzner D, et al. The burden of large and small duct primary sclerosing cholangitis in adults and children: a population-based analysis. Am J Gastroenterol 2007;102:1042-9.

78. Bergquist A, Lindberg G, Saarinen S, et al. Increased prevalence of primary sclerosing cholangitis among first-degree relatives. J Hepatol 2005;42:252-6.

79. Spurkland A, Saarinen S, Boberg KM, et al. HLA class II haplotypes in primary sclerosing cholangitis patients from five European populations. Tissue Antigens 1999;53:459-69.

80. Farrant JM, Doherty DG, Donaldson PT, et al. Amino acid substitutions at position 38 of the DR beta polypeptide confer susceptibility to and protection from primary sclerosing cholangitis. Hepatology 1992;16:390-5.

81. Mehal WZ, Lo YM, Wordsworth BP, et al. HLA DR4 is a marker for rapid disease progression in primary sclerosing cholangitis. Gastroenterology 1994;106:160-7.

82. Olerup O, Olsson R, Hultcrantz R, et al. HLA-DR and HLA-DQ are not markers for rapid disease progression in primary sclerosing cholangitis. Gastroenterology 1995;108:870-8.

83. Liu JZ, Hov JR, Folseraas T, et al. Dense genotyping of immunerelated disease regions identifies nine new risk loci for primary sclerosing cholangitis. Nat Genet 2013;45:670-5.

84. O'Mahony CA, Vierling JM. Etiopathogenesis of primary sclerosing cholangitis. Semin Liver Dis 2006; 26:3-21.

85. Palmer KR, Duerden BI, Holdsworth CD. Bacteriological and endotoxin studies in cases of ulcerative colitis submitted to surgery. Gut 1980;21:851-4.

86. Joo M, Abreu-e-Lima P, Farraye F, et al. Pathologic features of ulcerative colitis in patients with primary sclerosing cholangitis: a case-control study. Am J Surg Pathol 2009;33:854-62.

87. Sinakos E, Samuel S, Enders F, et al. inflammatory bowel disease in primary sclerosing cholangitis: a robust yet changing relationship. Inflamm Bowel Dis 2013;19:1004-9.

88. Jorgensen KK, Grzyb K, Lundin KE, et al. Inflammatory bowel disease in patients with primary sclerosing cholangitis: clinical characterization in liver transplanted and nontransplanted patients. Inflamm Bowel Dis 2012;18:536-45.

89. Verdonk RC, Dijkstra G, Haagsma EB, et al. Inflammatory bowel disease after liver transplantation: risk factors for recurrence and de novo disease. Am J Transplant 2006;6:1422-9.

90. Chapman RW, Arborgh BA, Rhodes JM, et al. Primary sclerosing cholangitis: a review of its clinical features, 
cholangiography, and hepatic histology. Gut 1980;21: 870-7.

91. Fevery J, Henckaerts L, Van Oirbeek R, et al. Malignancies and mortality in 200 patients with primary sclerosering cholangitis: a long-term single-centre study. Liver Int 2012;32:214-22.

92. Scheuer PJ. Ludwig Symposium on biliary disorders. Pathologic features and evolution of primary biliary cirrhosis and primary sclerosing cholangitis. Mayo Clinic Proc 1998;73:179-83.

93. Burak KW, Angulo P, Lindor KD. Is there a role for liver biopsy in primary sclerosing cholangitis? Am J Gastroenterol 2003;98:1155-8.

94. Stanich PP, Bjornsson E, Gossard AA, et al. Alkaline phosphatase normalization is associated with better prognosis in primary sclerosing cholangitis. Dig Liver Dis 2011;43:309-33.

95. Bjornsson E, Chari S, Silveira M, et al. Primary sclerosing cholangitis associated with elevated immunoglobulin G4: clinical characteristics and response to therapy. Am J Ther 2011;18:198-205.

96. Mendes FD, Jorgensen R, Keach J, et al. Elevated serum IgG4 concentration in patients with primary sclerosing cholangitis. Am J Gastroenterol 2006;101:2070-5.

97. Oseini AM, Chaiteerakij R, Shire AM, et al. Utility of serum immunoglobulin G4 in distinguishing immunoglobulin G4-associated cholangitis from cholangiocarcinoma. Hepatology 2011;54:940-8.

98. MacCarty RL, LaRusso NF, Wiesner RH, et al. Primary sclerosing cholangitis: findings on cholangiography and pancreatography. Radiology 1983;149:39-44.

99. Kaltenthaler E, Vergel YB, Chilcott J, et al. A systematic review and economic evaluation of magnetic resonance cholangiopancreatography compared with diagnostic endoscopic retrograde cholangiopancreatography. Health Technol Assess 2004;8:81-9.

100. Talwalkar JA, Angulo P, Johnson CD, et al. Cost-minimization analysis of MRC versus ERCP for the diagnosis of primary sclerosing cholangitis. Hepatology 2004;40:39-45.

101. Triantos CK, Koukias NM, Nikolopoulou VN, et al. Meta-analysis: ursodeoxycholic acid for primary sclerosing cholangitis. Aliment Pharmacol Ther 2011;54: 901-10.

102. Olsson R, Boberg KM, de Muckadell OS, et al. Highdose ursodeoxycholic acid in primary sclerosing cholangitis: a 5-year multicenter, randomized, controlled study. Gastroenterology 2005;129:1464-72.

103. Lindor KD, Kowdley KV, Luketic VA, et al. High-dose ursodeoxycholic acid for the treatment of primary sclerosing cholangitis. Hepatology 2009;50:808-14.

104. Eaton JE, Silveira MG, Pardi DS, et al. High-dose ursodeoxycholicacid is associated with the development of colorectal neoplasia in patients with ulcerative colitis and primary sclerosing cholangitis. Am J Gastroenterol 2011;106:1638-45.

105. Chapman R, Fevery J, Kalloo A, et al. Diagnosis and management of primary sclerosing cholangitis. Hepatology 2010;51:660-78.

106. Kaya M, Petersen BT, Angulo P, et al. Balloon dilation compared to stenting of dominant strictures in primary sclerosing cholangitis. Am J Gastroenterol 2001;96: 1059-66.
107. Washington MK. Autoimmune liver disease: overlap and outliers. Mod Path 2007;20:S15-30.

108. Burak KW, Urbanski SJ, Swain MG. A case of coexisting primary biliary cirrhosis and primary sclerosing cholangitis: a new overlap of autoimmune liver diseases. Dig Dis Sci 2001;46:2043-7.

109. Kingham JG, Abbasi A. Co-existence of primary biliary cirrhosis and primary sclerosing cholangitis: a rare overlap syndrome put in perspective. Eur J Gastroenterol Hepatol 2005; 17:1077-80.

110. Durazzo M, Premoli A, Paschetta E, et al. Overlap syndrome of autoimmune hepatitis: an open question. Dig Dis Sci 2013;58:344-8.

111. Czaja AJ. Frequency and nature of the variant forms of autoimmune liver disease. Hepatology 1998;28:360-5.

112. Salvio A, d'Errico T, Varriale M, et al. Overlap syndromes: more frequent forms between autoimmune liver diseases? IX National Meeting of the Federation of Associations of Hospital Doctors on Internal Medicine (FADOI), Rome, 2004.

113. Abdo AA, Bain VG, Kichian K, Lee SS. Evolution of autoimmune hepatitis in primary sclerosing cholangitis: a sequential syndrome. Hepatology 2002;36:1393-9.

114. Rust C, Beuers U. Overlap syndromes among autoimmune liver diseases. World J Gastroenterol 2008;14: 3368-73.

115. Woodward J, Neuberger J. Autoimmune overlap syndromes. Hepatology 2001;33:994-1002.

116. Chazouilleres O, Wendum D, Serfaty I, et al. Primary biliary cirrhosis-autoimmune hepatitis overlap syndrome: clinical features and response to therapy. Hepatology 1998;28:296-301.

117. European Association for the Study of the Liver. EASL Clinical Practice Guidelines: management of cholestatic liver diseases. J Hepatol 2009;51:237-67.

118. Floreani A, Rizzotto ER, Ferrara F, et al. Clinical course and outcome of autoimmune hepatitis/primary sclerosing cholangitis overlap syndrome. Am J Gastroenterol 2005; 100:1516-22.

119. Kaya M, Angulo P, Lindor KP. Overlap of autoimmune hepatitis and primary sclerosing cholangitis: an evaluation of a modified scoring system. J Hepatol 2000; 33:537-42.

120. Abdalian R, Dhar P, Jhaveri K, et al. Prevalence of sclerosing cholangitis in adults with autoimmune hepatitis: evaluating the role of routine magnetic resonance imaging. Hepatology 2008;47:949-57.

121. Wilshanski M, Chait P, Wade JA, et al. Primary sclerosing cholangitis in 32 children: clinical, laboratory and radiographic features with survival analyses. Hepatology 1995;22:1415-22.

122. Gregorio GV, Portmann B, Karani J, et al. Autoimmune hepatitis/sclerosing cholangitis overlap syndrome in childhood: a 16-year prospective study. Hepatology 2001;33:544-53.

123. Trivedi PJ, Hirschfield GM. Review article: overlap syndromes and autoimmune liver disease. Aliment Pharmacol Ther 2012;36:517-33.

124. Miyake Y, Iwasaki Y, Kobashi H, et al. Efficacy of ursodeoxycholic acid for Japanese patients with autoimmune hepatitis. Hepatol Int 2009;3:556-62.

125. Joshi S, Cauch-Dudek K, Wanless IR, et al. Primary biliary cirrhosis with additional features of autoimmune 
hepatitis: response to therapy with ursodeoxycholic acid. Hepatology 2002;35:409-13.

126. Boberg KM, Chapman RW, Hirschfield GM, et al. Overlap syndromes: the international autoimmune hepatitis group (IAIHG) position statement on a controversial issue. J Hepatol 2011;54:374-85.

127. Chazouilleres O, Wendum D, Serfaty L, et al. Long term outcome and response to therapy of primary biliary cirrhosis-autoimmune hepatitis overlap syndrome. J Hepatol 2006;44:400-6.

128. Al-Chalabi T, Portmann BC, Bernal W, et al. Autoimmune hepatitis overlap syndromes: an evaluation of treatment response, long-term outcome and survival. Aliment Pharmacol Ther 2008;28:209-20.

129. Mieli-Vergani G, Heller S, Jara P, et al. Autoimmune hepatitis. J Pediatr Gastroenterol Nutr 2009;49:158-64.

130. Feldstein AE, Perrault J, El-Youssif M, et al. Primary sclerosing cholangitis in children: a long-term followup study. Hepatology 2003;38:210-7.
131. Saich R, Chapman R. Primary sclerosing cholangitis, autoimmune hepatitis and overlap syndromes in inflammatory bowel disease. World J Gastroenterol 2008;14: 331-7.

132. Mitchell SA, Bansi DS, Hunt N, et al. A preliminary trial of high dose ursodeoxycholic acid in primary sclerosing cholangitis. Gastroenterology 2001;121:900-7.

133. Lindstrom L, Boberg KM, Wikman O, et al. High dose ursodeoxycholic acid in primary sclerosing cholangitis does not prevent colorectal neoplasia. Aliment Pharmacol Ther 2012;35:451-7.

134. Lüth S, Kanzler S, Frenzel C, et al. Characteristics and long-term prognosis of the autoimmune hepatitis/primary sclerosing cholangitis overlap syndrome. J Clin Gastroenterol 2009;43:75-80.

135. Perdigoto R, Carpenter HA, Czaja AJ. Frequency and significance of chronic ulcerative colitis in severe corticosteroid-treated autoimmune hepatitis. J Hepatol 1992;14:325-31. 